\title{
PSR J1024-0719: A MILLISECOND PULSAR IN AN UNUSUAL LONG-PERIOD ORBIT
}

\author{
David L. Kaplan ${ }^{1}$, Thomas Kupfer ${ }^{2}$, David J. Nice ${ }^{3}$, Andreas Irrgang ${ }^{4}$, Ulrich Heber ${ }^{4}$, Zaven Arzoumanian ${ }^{5}$, \\ Elif Beklen ${ }^{6,7}$, Kathryn Crowter ${ }^{8}$, Megan E. DeCesar ${ }^{1}$, Paul B. Demorest ${ }^{9}$, Timothy Dolch ${ }^{10}$, Justin A. Ellis ${ }^{11}$, \\ Robert D. Ferdman ${ }^{12}$, Elizabeth C. Ferrara ${ }^{13}$, Emmanuel Fonseca ${ }^{8}$, Peter A. Gentile ${ }^{7}$, Glenn Jones ${ }^{14}$, \\ Megan L. Jones ${ }^{7}$, Simon Kreuzer ${ }^{4}$, Michael T. Lam ${ }^{15}$, Lina Levin ${ }^{7}$, Duncan R. Lorimer ${ }^{7}$, Ryan S. Lynch ${ }^{16}$, \\ Maura A. Mclaughlin ${ }^{7}$, Adam A. Miller ${ }^{2,11,23}$, Cherry NG ${ }^{8}$, Timothy T. Pennucci ${ }^{17}$, Tom A. Prince ${ }^{2}$, Scott M. Ransom ${ }^{18}$, \\ Paul S. Ray ${ }^{19}$, Renee Spiewak ${ }^{1}$, Ingrid H. Stairs ${ }^{8,20}$, Kevin Stovall ${ }^{21}$, JosePh Swiggum ${ }^{1}$, and Weiwei Zhu ${ }^{22}$ \\ ${ }^{1}$ Center for Gravitation, Cosmology and Astrophysics, Department of Physics, University of Wisconsin-Milwaukee, P.O. Box 413, Milwaukee, WI 53201, USA \\ ${ }^{2}$ Division of Physics, Mathematics, and Astronomy, California Institute of Technology, Pasadena, CA 91125, USA \\ ${ }^{3}$ Department of Physics, Lafayette College, Easton, PA 18042, USA \\ ${ }^{4}$ Dr. Karl Remeis-Observatory \& ECAP, Astronomical Institute, Friedrich-Alexander University Erlangen-Nuremberg, Sternwartstrasse 7, D-96049 Bamberg, \\ Germany \\ ${ }^{5}$ Center for Research and Exploration in Space Science and Technology and X-Ray Astrophysics Laboratory, NASA Goddard Space Flight Center, Code 662, \\ Greenbelt, MD 20771, USA \\ ${ }^{6}$ Department of Physics, Suleyman Demirel University, 32260, Isparta, Turkey \\ ${ }^{7}$ Department of Physics and Astronomy, West Virginia University, P.O. Box 6315, Morgantown, WV 26506, USA \\ ${ }^{8}$ Department of Physics and Astronomy, University of British Columbia, 6224 Agricultural Road, Vancouver, BC V6T 1Z1, Canada \\ ${ }^{9}$ National Radio Astronomy Observatory, 1003 Lopezville Road, Socorro, NM 87801, USA \\ ${ }^{10}$ Department of Physics, Hillsdale College, 33 E. College Street, Hillsdale, Michigan 49242, USA \\ ${ }^{11}$ Jet Propulsion Laboratory, California Institute of Technology, 4800 Oak Grove Drive, Pasadena, CA 91109, USA \\ ${ }^{12}$ Department of Physics, McGill University, 3600 University Street, Montreal, QC H3A 2T8, Canada \\ ${ }^{13}$ NASA Goddard Space Flight Center, Greenbelt, MD 20771, USA \\ ${ }^{14}$ Department of Physics, Columbia University, New York, NY 10027, USA \\ ${ }^{15}$ Department of Astronomy, Cornell University, Ithaca, NY 14853, USA \\ ${ }^{16}$ National Radio Astronomy Observatory, P.O. Box 2, Green Bank, WV 24944, USA \\ ${ }^{17}$ University of Virginia, Department of Astronomy, P.O. Box 400325, Charlottesville, VA 22904, USA \\ ${ }^{18}$ National Radio Astronomy Observatory, 520 Edgemont Road, Charlottesville, VA 22903, USA \\ ${ }^{19}$ Space Science Division, Naval Research Laboratory, Washington, DC 20375-5352, USA \\ ${ }^{20}$ McGill Space Institute, 3550 Rue University, Montreal, Quebec H3A 2A7 Canada \\ ${ }^{21}$ Department of Physics and Astronomy, University of New Mexico, Albuquerque, NM 87131, USA \\ ${ }^{22}$ Max Planck Institute for Radio Astronomy, Auf dem Hügel 69, D-53121 Bonn, Germany \\ Received 2016 March 31; accepted 2016 May 10; published 2016 July 25
}

\begin{abstract}
PSR J1024-0719 is a millisecond pulsar that was long thought to be isolated. However, puzzling results concerning its velocity, distance, and low rotational period derivative have led to a reexamination of its properties. We present updated radio timing observations along with new and archival optical data which show that PSR $\mathrm{J} 1024-0719$ is most likely in a long-period $(2-20 \mathrm{kyr})$ binary system with a low-mass $\left(\approx 0.4 M_{\odot}\right)$, low-metallicity $(Z \approx-0.9$ dex $)$ main-sequence star. Such a system can explain most of the anomalous properties of this pulsar. We suggest that this system formed through a dynamical exchange in a globular cluster that ejected it into a halo orbit, which is consistent with the low observed metallicity for the stellar companion. Further astrometric and radio timing observations such as measurement of the third period derivative could strongly constrain the range of orbital parameters.
\end{abstract}

Key words: binaries: general - pulsars: individual (PSR J1024-0719) - stars: distances

\section{INTRODUCTION}

\subsection{Pulsar Characteristics and Early Distance Estimates}

PSR J1024-0719 is a millisecond pulsar (MSP) with a rotation period of $P=5.2 \mathrm{~ms}$ and a period derivative of $\dot{P}=1.8 \times 10^{-20}$ (Bailes et al. 1997), typical of other MSPs. There was no evidence for binary motion in timing observations of the pulsar, and so it was regarded as isolated. Its dispersion measure, $\mathrm{DM}=6.5 \mathrm{pc} \mathrm{cm}^{-3}$, is among the lowest measured; it implies a distance of $d_{\mathrm{DM}} \approx 0.390 \mathrm{kpc}$ based on the Cordes \& Lazio (2002) Galactic electron density model.

A second line of reasoning also led to similar distance estimates. The observed pulsar period derivatives $\dot{P}_{\mathrm{obs}}$ are biased from their intrinsic values $\dot{P}_{\text {int }}$ according to the Shklovskii effect, $\dot{P}_{\text {obs }}=\dot{P}_{\text {int }}+\dot{P}_{\text {Shk }}$ with $\dot{P}_{\text {Shk }}=\mu^{2} d P / c$,

\footnotetext{
${ }^{23}$ Hubble Fellow.
}

where $\mu$ is the proper motion, $d$ is the distance, and $c$ is the speed of light (Shklovskii 1970). For a pulsar losing rotational energy, the intrinsic spin-down rate must be positive, $\dot{P}_{\text {int }}>0$, so that the Shklovskii effect places an upper limit on the distance, $d_{\dot{P}}<\dot{P}_{\text {obs }} c / \mu^{2} P$. Toscano et al. (1999) used an early

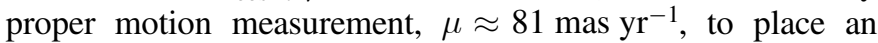
upper limit of $d_{\dot{P}}<0.226 \mathrm{kpc}$. Later measurements revised the proper motion down to $\mu \approx 60$ mas which gives $d_{\dot{P}}<0.430 \mathrm{kpc}$, consistent with $d_{\mathrm{DM}}$ (Hotan et al. 2006).

\subsection{Optical and $\gamma$-ray Observations}

Sutaria et al. (2003) searched for an optical counterpart to PSR J1024-0719 using deep observations with the Very Large Telescope (VLT). They found two potential counterparts: a faint one (1024-Fnt, with $R=24.4$ ) and a bright one, which appeared to be a $\mathrm{K}$ star $(1024-\mathrm{Br}$, with $R=18.9)$. While they 
Table 1

Parallax and Distance Measurements for PSR J1024-0719

\begin{tabular}{|c|c|c|c|}
\hline Type & $\begin{array}{c}\text { Parallax } \\
\text { (mas) }\end{array}$ & $\begin{array}{c}\text { Distance } \\
(\mathrm{kpc})\end{array}$ & Reference \\
\hline Dispersion Measure & $\ldots$ & 0.39 & Cordes \& Lazio (2002) \\
\hline$\dot{P}$ limit & $\ldots$ & $<0.43$ & Hotan et al. (2006) \\
\hline PPTA $^{\mathrm{b}}$ Timing & $0.5 \pm 0.3$ & $1.1_{-0.3}^{+0.4 \mathrm{~d}}$ & Reardon et al. (2016) \\
\hline Nançay Timing & $0.89 \pm 0.14$ & $1.13 \pm 0.18$ & Guillemot et al. (2016) \\
\hline EPTA $^{\mathrm{c}}$ Timing & $0.80 \pm 0.17$ & $1.08_{-0.16}^{+0.23 \mathrm{~d}}$ & Desvignes et al. (2016) \\
\hline
\end{tabular}

Notes.

a North American Nanohertz Observatory for Gravitational waves, http://www.nanograv.org.

b Parkes Pulsar Timing Array, http://www.atnf.csiro.au/research/pulsar/ppta/.

${ }^{\mathrm{c}}$ European Pulsar Timing Array, http://www.epta.eu.org.

d Includes adjustment for Lutz-Kelker bias (Verbiest et al. 2012).

${ }^{\mathrm{e}}$ For an assumed companion mass of $0.4 M_{\odot}$.

found the position of 1024-Br to be coincident with that of the pulsar to within $0 . \prime 2$, they rejected an association between $1024-\mathrm{Br}$ and the pulsar because (i) $1024-\mathrm{Br}$ is much more distant than the distance estimate for the pulsar then available; (ii) the proper motion of $1024-\mathrm{Br}$, which they estimated by comparing their observations with earlier catalogs, disagreed with the proper motion of the pulsar reported by Toscano et al. (1999); and (iii) the very small timing residuals of the pulsar suggested that it was an isolated object, with no evidence for binary motion.

Espinoza et al. (2013) identified a Fermi $\gamma$-ray counterpart to PSR J1024-0719. They assumed that the pulsar was at a distance of $d=0.4 \mathrm{kpc}$, which is close to the maximum allowed by the Shklovskii effect (they used $d<0.410 \mathrm{kpc}$ ). Correcting for the Shklovskii effect, they estimated the intrinsic period derivative to be $\dot{P}_{\text {int }} \leqslant 5 \times 10^{-22}$, an unusually small value which implies an unusually small rotational energy loss rate, $\dot{E} \propto \dot{P} / P^{3}$. This, in turn, yielded a value for the $\gamma$-ray efficiency, $\eta=L_{\gamma} / \dot{E}>0.8$, that is higher than typically found.

\subsection{Recent Developments}

PSR J1024-0719 is under observation by several groups as part of the global effort to detect nanohertz gravitational waves via MSP timing.

Four recent papers used high-precision timing to measure or constrain its parallax, and hence its distance; we summarize these measurements in Table 1. All of these recent pulsar timing distances are consistent with each other, and they are incompatible with the distance upper limit $d_{\dot{P}}<0.430 \mathrm{kpc}$ derived from the Shklovskii effect using the latest proper motion measurements (e.g., Matthews et al. 2016). Three of these papers argued that the discrepancy between these distances could be resolved if the pulsar were undergoing acceleration, $\dot{v}$, due to gravitational interaction with another object (Matthews et al. 2016; Guillemot et al. 2016; Desvignes et al. 2016). This would add a further bias to the observed spindown rate, $\dot{P}_{\text {obs }}=\dot{P}_{\text {int }}+\dot{P}_{\text {Shk }}+\dot{P}_{\text {orb }}$, where $\dot{P}_{\text {orb }}=\dot{v} P / c$. If the pulsar were undergoing negative acceleration, $\dot{v}<0$, then the resulting negative $\dot{P}_{\text {orb }}$ term would allow for a larger positive $\dot{P}_{\text {Shk }}=\mu^{2} d / c$ term, which in turn would allow it to contain a larger distance than the previous upper limit of $0.430 \mathrm{kpc}$.

Such gravitational interaction (i.e., an orbit) could potentially be manifested in timing observations as a rotation period second derivative, $\ddot{P}$, due to the jerk, or change in acceleration, of the pulsar (details discussed below). Matthews et al. (2016) combined their data with previous observations and placed an upper limit of $\ddot{P} \lesssim 1 \times 10^{-23} \mathrm{yr}^{-1}=3 \times 10^{-31} \mathrm{~s}^{-1}$. Considering this and other constraints, they found that the pulsar acceleration could be caused by an orbit with period greater than about 14,000 years and a companion star mass of $0.1 M_{\odot}$ or greater, and they noted that $1024-\mathrm{Br}$ satisfied this mass constraint.

Guillemot et al. (2016) measured $\ddot{P}=(2.2 \pm 0.2) \times$ $10^{-24} \mathrm{yr}^{-1}=(7.0 \pm 0.6) \times 10^{-32} \mathrm{~s}^{-1}$, a value just under the limit of Matthews et al. (2016). Reardon et al. (2016) and Desvignes et al. (2016) reported red timing noise (see also Caballero et al. 2015); such red noise could be indicative of a nonzero value of $\ddot{P}$ that was not accounted for in the timing model applied to their data.

\subsection{This Paper}

In this paper, we argue that PSR J1024-0719 is in a longperiod (2-20 kyr) binary system with 1024-Br. In Section 2, we present updated NANOGrav timing observations of PSR J1024-0719, including new parallax and period second derivative measurements. In Section 3, we present an astrometric analysis from new and archival optical data for 1024-Br and show that its position and proper motion are completely consistent with those of the pulsar, leaving no doubt that they are a common proper motion pair. Additionally, we present a spectroscopic analysis of $1024-\mathrm{Br}$ and show that the companion is consistent with a star of spectral type $\mathrm{K}$ or M. In Section 4, we use constraints on the position offset, acceleration, and jerk in this system to analyze the possible binary system parameters for both circular and generalized orbits. We find the binary to be very wide and the pulsar space velocity to be unusually fast. In Section 5, we discuss formation scenarios for such a system. In Section 6, we summarize our results. 
Unless otherwise noted, proper motions in right ascension $\alpha$ are $\mu_{\alpha}=\dot{\alpha} \cos \delta$ in units of mas $\mathrm{yr}^{-1}$, and all positions are J2000.

During the preparation of this paper, we became aware that another group had come to similar conclusions regarding the nature of PSR J1024-0719 (Bassa et al. 2016). Our analysis is very similar to that presented in Bassa et al. (2016), although our data (aside from archival optical observations) are entirely independent. Bassa et al. (2016) additionally present an alternate formation mechanism for PSR J1024-0719 which we discuss briefly in Section 5.

\section{RADIO TIMING}

We conducted radio timing observations of PSR J1024-0719 over 6.3 years using the $100 \mathrm{~m}$ Robert C. Byrd Green Bank Telescope. The resulting data will be part of the upcoming NANOGrav 11 year data set (Z. Arzoumanian et al. 2016, in preparation). The observation and data-reduction procedures are nearly identical to those of the NANOGrav 9 year data set (Arzoumanian et al. 2015). Briefly, pulse arrival times were determined using two separate receiver systems, near 820 and $1400 \mathrm{MHz}$, at roughly monthly intervals. The arrival times were fit to a standard timing model using the TEMPO package. ${ }^{24}$ The timing model included the following: astrometric parameters; independent dispersion measure at every epoch (where an epoch is defined as a period of six days or less); a white noise model; a pulsar frequency model as described below. The JPL DE430 Solar System ephemeris (Folkner et al. 2014) was used for Earth's motion around the solar system, and so astrometric values are relative to this frame, which in turn is tied to the Second Realization of the International Celestial Reference Frame (ICRF2). The ephemeris was rotated by $23^{\circ} 26^{\prime} 21^{\prime \prime} 406$, that is, the IERS2010 obliquity of the ecliptic, to provide the position and proper motion in ecliptic coordinates. Arrival times were adjusted following the TT(BIPM15) ${ }^{25}$ timescale, and parameters are ultimately presented in Barycentric Dynamical Time. Besides using updated ephemeris and time standards, the primary difference between our work and the analysis procedure of Arzoumanian et al. (2015) is our use of frequency derivatives, as described below, instead of a red noise model.

Best-fit timing model parameters are given in Table 2. The residual pulse arrival times after subtracting off the timing model, and the variation in DM over time, are shown in Figure 1. The two most important results from the timing analysis are (i) a new measurement of the pulsar parallax and (ii) a significant measurement of the rotation second frequency derivative.

The new parallax measurement is $\varpi=0.77 \pm 0.23$ mas, corresponding to a distance of $1.3_{-0.3}^{+0.6} \mathrm{kpc}$. This agrees with other recent measurements given in Table 1 . We checked our distance measurement against the Lutz-Kelker bias-estimate code of Verbiest et al. (2012) ${ }^{26}$ and found that the distance estimate changed by less than $1 \sigma$; we elected not to include this in our reported distance measurement. In the analysis below, we use our parallax measurement, despite there being values with nominally smaller uncertainties in the literature, as we believe that our dispersion modeling algorithm (fitting

\footnotetext{
${ }^{24}$ http://tempo.sourceforge.net

$25 \mathrm{ftp}$ ///tai.bipm.org/TFG/TT(BIPM)

${ }^{26}$ http://psrpop.phys.wvu.edu/LKbias/
}

Table 2

Timing Parameters of PSR J1024-0719

\begin{tabular}{lc}
\hline \hline & Data Set \\
\hline MJD range & $55100-57378$ \\
Data span (years) & 6.3 \\
Number of TOAs & 8501 \\
Number of Epochs & 83 \\
\hline
\end{tabular}

Timing Parameters

\begin{tabular}{lc}
\hline Ecliptic longitude, $\lambda$ (deg.) & $160.734351327(14)$ \\
Ecliptic latitude, $\beta$ (deg.) & $-16.04472741(6)$ \\
Proper motion in $\lambda, \mu_{\lambda}=\dot{\lambda} \cos \beta\left(\mathrm{mas} \mathrm{yr}^{-1}\right)$ & $-14.37(3)$ \\
Proper motion in $\beta, \mu_{\beta}=\dot{\beta}\left(\operatorname{mas~yr}^{-1}\right)$ & $-57.97(13)$ \\
Parallax, $\varpi$ (mas) & $0.77(23)$ \\
Rotation frequency, $f_{0}\left(\mathrm{~s}^{-1}\right)$ & $193.7156863778085(8)$ \\
Rotation frequency first derivative, $f_{1}\left(\mathrm{~s}^{-2}\right)$ & $-6.9638(4) \times 10^{-16}$ \\
Rotation frequency second derivative, $f_{2}\left(\mathrm{~s}^{-3}\right)$ & $-4.1(10) \times 10^{-27}$ \\
Rotation frequency third derivative, $f_{3}\left(\mathrm{~s}^{-4}\right)$ & $1.1(7) \times 10^{-34}$ \\
Epoch of period and position (MJD) & 56236.000
\end{tabular}

\begin{tabular}{lc}
\hline \multicolumn{2}{c}{ Derived Quantities } \\
\hline Right ascension, $\alpha(\mathrm{J} 2000)$ & $10^{\mathrm{h} 24^{\mathrm{m}} 38^{\mathrm{s}} .667384(7)}$ \\
Declination, $\delta(\mathrm{J} 2000)$ & $-07^{\circ} 19^{\prime} 19^{\prime \prime} 5970(2)$ \\
Proper motion in $\alpha, \mu_{\alpha}=\dot{\alpha} \cos \delta\left(\mathrm{mas} \mathrm{yr}^{-1}\right)$ & $-35.26(6)$ \\
Proper motion in $\delta, \mu_{\delta}=\dot{\delta}\left(\mathrm{mas} \mathrm{yr}^{-1}\right)$ & $-48.21(13)$ \\
Total proper motion, $\mu\left(\mathrm{mas} \mathrm{yr}^{-1}\right)$ & $59.73(13)$ \\
Period, $P(\mathrm{~s})$ & $0.00516220456225561(2)$ \\
Period first derivative, $\dot{P}\left(\mathrm{~s} \mathrm{~s}^{-1}\right)$ & $1.85575(12) \times 10^{-20}$ \\
Period second derivative, $\ddot{P}\left(\mathrm{~s}^{-1}\right)$ & $1.1(3) \times 10^{-31}$ \\
Period third derivative, $\dddot{P}\left(\mathrm{~s}^{-2}\right)$ & $-3(2) \times 10^{-39}$ \\
Distance, $d_{\varpi}(\mathrm{kpc})$ & $1.3_{-0.3}^{+0.6}$ \\
\hline
\end{tabular}

Note.

${ }^{\mathrm{a}}$ Numbers in parentheses are $1 \sigma$ uncertainties in the last digit quoted.

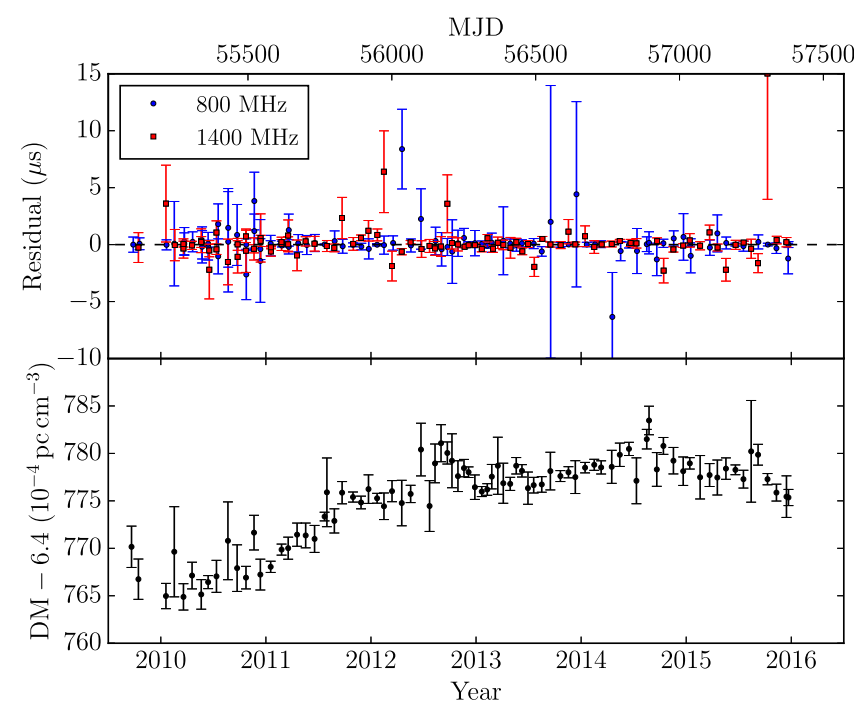

Figure 1. Upper panel: residual pulse arrival times after removing the best-fit timing model. Points represent daily averages for a given receiver; blue circles are $800 \mathrm{MHz}$ and red squares are $1400 \mathrm{MHz}$. Lower panel: dispersion measure at every epoch. These values were fit simultaneously with all other timing parameters.

independent dispersion measures at every observing epoch) yields more robust measurements, especially for pulsars such as PSR J1024-0719 which lie at low ecliptic latitudes (Matthews 
et al. 2016). Adopting other parallax results would not qualitatively change our analysis.

In the timing model, we parametrized the pulsar spin by the pulsar rotation frequency $f_{0}$ and three frequency derivatives $\left(f_{1} \equiv d f / d t, f_{2} \equiv d^{2} f / d t^{2}\right.$, and $\left.f_{3} \equiv d^{3} f / d t^{3}\right)$. The measured values of these frequency derivatives, and the corresponding values for pulse period and its derivatives, are listed in Table 2. Since $f_{3}$ (or, equivalently, $\dddot{P}$ ) is of potential interest, we left it in the timing solution even though its measurement is not formally significant.

We measured a significant rotation frequency second derivative, $f_{2}=(-4.1 \pm 1.0) \times 10^{-27} \mathrm{~s}^{-3}$. This could arise due to binary motion or due to noise in the rotation of the pulsar ("timing noise"). To check for the latter possibility, we compare our observations with a scaling law for timing noise developed by Shannon \& Cordes (2010). We use their model which incorporated canonical pulsars, MSPs, and magnetars. Given the $f_{0}$ and $f_{1}$ of PSR J1024-0719, and considering the time span of our observations, their model predicts excess residuals of $0.06 \mu \mathrm{s}$, albeit with large uncertainty. We estimate that our measured $f_{2}$ would contribute $0.40 \mu$ s if not included in the timing model, i.e., substantially more than the noise model prediction. Therefore, it is unlikely, though not impossible, that the observed $f_{2}$ is due to timing noise. For the remainder of this paper, we interpret $f_{2}$ as the jerk, or the change in acceleration, of the pulsar due to binary motion.

The measured $f_{2}$ is equivalent to period second derivative $\ddot{P}=(1.1 \pm 0.3) \times 10^{-31} \mathrm{~s}^{-1}$. This is in agreement with the value of $(0.70 \pm 0.06) \times 10^{-31} \mathrm{~s}^{-1}$ reported by Guillemot et al. (2016). Our measurement uncertainty is relatively large due to covariance between $f_{2}$ and variations in interstellar DM, which we fit independently at every epoch simultaneously with the other parameters; in contrast, Guillemot et al. (2016) used a linear model in DM which was held fixed in their final timing solutions. In the presence of significant DM variations (Figure 1), we believe our method yields the most robust values of $f_{2}$ or $\ddot{P}$. This same reasoning applies to our (nonsignificant) measurement of $f_{3}=(1.1 \pm 0.7) \times 10^{-34} \mathrm{~s}^{-4}$. For example, changing the nature of the DM fit in our 6 year long data set from a constant value, to a polynomial of degree up to 7 , or to the by-epoch fit given in Table 2, changes $f_{3}$ by few $\times 10^{-35} \mathrm{~s}^{-4}$. Given that the $f_{3}$ fit depends on time to the fourth power, this will be even more apparent in longer data sets.

\section{OPTICAL OBSERVATIONS AND ANALYSIS}

\subsection{Optical Imaging}

We obtained images of the field around PSR J1024-0719 with the Palomar 60 inch telescope (P60; Cenko et al. 2006). The data consist of $4 \times 120 \mathrm{~s}$ exposures in the $r^{\prime}$ band on the night of 2016 January 16 dithered by about $20^{\prime \prime}$ each. The data were processed through the standard P60 pipeline, which determined independent astrometry and photometric solutions for each image using the USNO B-1.0 catalog. The pipeline is described in full detail in Cenko et al. (2006).

\subsection{Absolute Astrometry}

In Figure 2, we show the position of PSR J1024-0719 (Table 2) corrected to the epoch of the P60 images (MJD 57403). This position is 0."03 from the position of 1024-Br, which we compare with a typical absolute astrometric

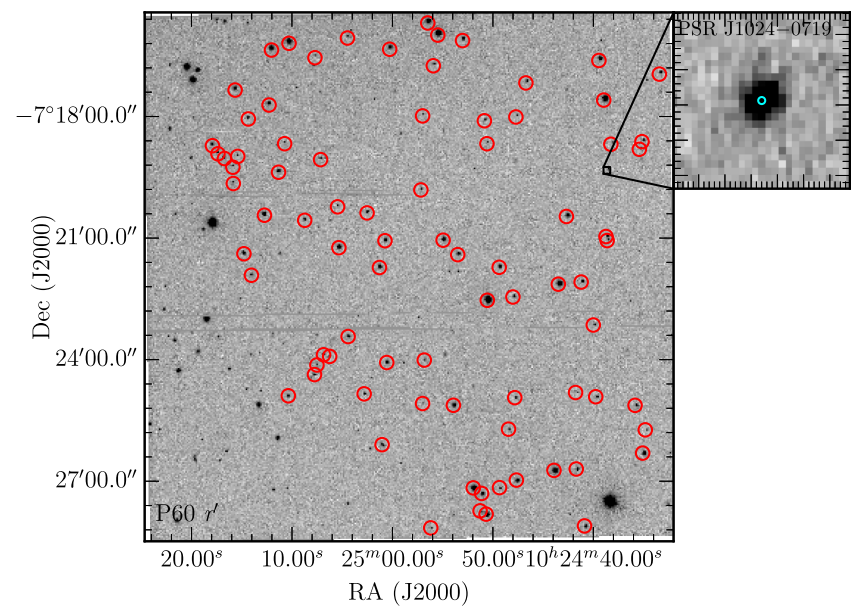

Figure 2. P60 $r^{\prime}$ image of PSR J1024-0719 from 2016 January 16. This is a single $120 \mathrm{~s}$ exposure. The 2MASS reference stars used for astrometry are circled. The inset box shows the region around the radio position of PSR J1024-0719 (Table 2) corrected to the epoch of the P60 image shown by the cyan circle. The radius of that circle is $0 . / 2$, which is the typical absolute astrometric uncertainty of the P60 astrometry.

uncertainty of 0. . 2 for the P60 pipeline. Likewise, the propermotion-corrected pulsar position is 0." 11 away from Two Micron All-Sky Survey (2MASS; Skrutskie et al. 2006) source 2MASS 10243869-0719190. We assess the false association rate of the 2MASS source with PSR J1024-0719 by considering that within $1^{\circ}$ of PSR J1024-0719, there are 7500 2MASS sources brighter than 2MASS 102438690719190 for an areal density of $(1.84 \pm 0.02) \times 10^{-4} \operatorname{arcsec}^{-2}$. Therefore, the association rate is about $1.3 \times 10^{-5}$ and we can be quite confident that the pulsar is associated with 1024-Br/2MASS 10243869-0719190.

We further verify the astrometry by noting that the J2000 position of the pulsar is 0." 03 away from PPMXL (Roeser et al. 2010) source 3714292468260686336, and 0.' 15 away from Absolute Proper Motions Outside the Plane (APOP; Qi et al. 2015) source APOP 39332+0000404, all of which are consistent with 1024-Br (see Table 3). For 2MASS and PPMXL, the proposed counterpart is within $1 \sigma$ of the proper motion-corrected radio timing position. In APOP, the proposed counterpart is slightly further away and the quoted accuracy of APOP of \pm 0.2 mas relative to the ICRF suggests that the offset, 0 ." $15 \pm 0$." 03 , may be significant, but we are cautious with frame ties between the radio and optical systems (e.g., Vickers et al. 2016), and so in what follows we largely treat this as an upper limit to the projected separation.

\subsection{Relative Astrometry and Proper Motions}

To determine the proper motions of the stars in this field, we compared our P60 observations against the 2MASS Point Source Catalog (PSC). We measured the positions of all of the stars in the P60 images using sextractor (Bertin \& Arnouts 1996) and matched each exposure separately to the 2MASS sources. We required that the source be $<3$ " from its 2MASS position and that it have no quality flags suggesting questionable data (bad pixels, saturation, etc.), and found 82 reference sources over the P60 image plus the possible counterpart to the pulsar (which is itself a 2MASS source as discussed above; see Figure 2). We then computed position offsets between the positions measured in the P60 images 
Table 3

Astrometry of PSR J1024-0719 and its Optical Counterpart

\begin{tabular}{|c|c|c|c|c|c|}
\hline Survey & $\begin{array}{c}\Delta \alpha^{\mathrm{a}} \\
(\operatorname{arcsec})\end{array}$ & $\begin{array}{c}\Delta \delta^{\mathrm{a}} \\
(\operatorname{arcsec})\end{array}$ & $\begin{array}{c}\mu_{\alpha} \\
\left(\operatorname{mas~yr}^{-1}\right)\end{array}$ & $\begin{array}{c}\mu_{\delta} \\
\left(\operatorname{mas~yr}^{-1}\right)\end{array}$ & Reference \\
\hline NANOGrav & 0 & 0 & $-35.26 \pm 0.06$ & $-48.21 \pm 0.13$ & This paper \\
\hline P60-2MASS & $0.03 \pm 0.20$ & $-0.11 \pm 0.20$ & $-33.9 \pm 6.5$ & $-43.4 \pm 6.5$ & Skrutskie et al. (2006)/this paper \\
\hline APOP & $-0.11 \pm 0.04$ & $0.09 \pm 0.03$ & $-33.5 \pm 1.9$ & $-51.7 \pm 1.2$ & Qi et al. (2015) \\
\hline PPMXL & $0.00 \pm 0.11$ & $0.03 \pm 0.11$ & $-29.0 \pm 7.0$ & $-44.2 \pm 7.0$ & Roeser et al. $(2010)^{\mathrm{b}}$ \\
\hline
\end{tabular}

Notes.

${ }^{\text {a }}$ Offsets between the optical counterpart and the radio position of PSR J1024-0719 at epoch 2000.0

b PPMXL proper motions have been corrected according to Vickers et al. (2016).

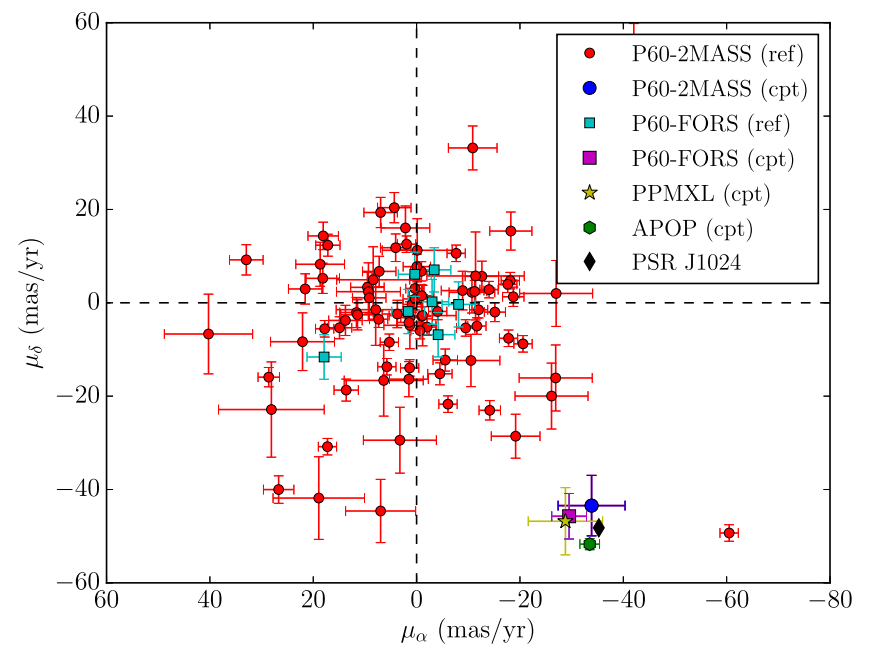

Figure 3. Proper motions for the stars in the PSR J1024-0719 field. The circles are for the P60 data compared to the 2MASS Point Source Catalog, with the red circles the reference sources and the larger green circle the putative counterpart to PSR J1024-0719 identified by Sutaria et al. (2003). The squares are for the P60 data compared to the archival VLT/FORS1 data, with the cyan squares the reference sources and the larger magenta square the putative counterpart. The yellow star is the proper motion of source 3714292468260686336 from the PPMXL catalog (Roeser et al. 2010), and the cyan hexagon the source $39332+0000404$ from the APOP catalog (Qi et al. 2015), both of which are within 0". 15 of PSR J1024-0719 and consistent with the candidate counterpart 1024-Br. The proper motion of PSR J1024-0719 measured in Section 2 is the black diamond.

(MJD 57403) and the 2MASS PSC (MJD 51193), which we show in Figure 3.

The majority of the reference stars had proper motions with

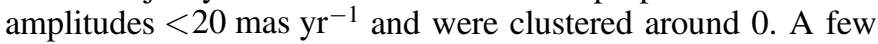
stars had individually significant proper motions, among them 1024-Br. We find a proper motion for this star of

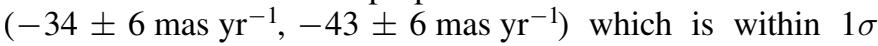
of our measurement of the pulsar proper motion (Table 2).

We verified this proper motion using the same imaging data used by Sutaria et al. (2003). We retrieved data taken by the ESO $8.2 \mathrm{~m}$ Very Large Telescope Antu (VLT-UT1) with the FORS1 CCD in the narrow-field imaging mode in the Bessel $V$ band (the other bands were similar) from the ESO archive, finding $3 \times 120 \mathrm{~s}$ exposures. We reduced the data using custom routines, removing the overscan, subtracting a bias frame, and then flat-fielding the images. The much narrower field of view (205" versus $\left.774^{\prime \prime}\right)$ and the much larger telescope mean that many fewer reference stars were available, with only seven sources that we could match to our P60 data. We determined the position offset of all of the sources in the FORS1 data (MJD 51996) compared to the P60 data, averaging over the individual exposures in both data sets. We find a proper motion for 2MASS J10243869-0719190 of

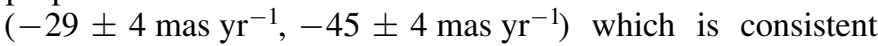
with both our measurement from P60 to 2MASS as well as the NANOGrav proper motion (Figure 3). There were insufficient sources that matched between the FORS1 data and 2MASS for a third proper motion measurement, as well as a significantly reduced time baseline ( 800 days, versus $15-17$ years).

Finally, the proper motions of the radio pulsar and the APOP and PPMXL sources (Table 3 and Figure 3) are all consistent to within the uncertainties. We conclude that PSR J1024-0719 and 1024-Br form a common proper motion pair.

\subsection{Spectral Analysis}

Optical spectra of 1024-Br were obtained with the Palomar 200 inch telescope and the Double Beam Spectrograph (DBSP; Oke \& Gunn 1982) on 2016 January 30 using a low-resolution mode $(R \sim 1500)$. We took three exposures with an exposure time of $1000 \mathrm{~s}$ each. Both arms of the spectrograph were reduced using a custom PyRAF-based pipeline. ${ }^{27}$ The pipeline performs standard image processing and spectral reduction procedures, including bias subtraction, flat-field correction, wavelength calibration, optimal spectral extraction (Horne 1986), and flux calibration. For the analysis, all three individual exposures were combined resulting in a signal-tonoise ratio $(\mathrm{S} / \mathrm{N})$ of about 25 at $7000 \AA$.

We fit the red part of the normalized spectrum using Phoenix models (Husser et al. 2013), which are multiplied with a telluric transmission spectrum (Moehler et al. 2014) to account for telluric absorption. The region around the $\mathrm{Na}$ I doublet (5889.961-5895.924 A) was ignored because of contamination with night sky emission lines. The telluric absorption bands were used to correct the wavelength scale for instrument flexure. The fitting parameters included the radial velocity $v_{r}$, the effective temperature $T_{\text {eff }}$, and the metallicity $Z$. Since spectroscopic determination of surface gravities for cool stars is notoriously difficult even from high-resolution, high-fidelity spectra (Smiljanic et al. 2014), we kept the surface gravity fixed at $\log g=4.9$ dex (see Section 3.5). We found a good fit (Figure 4) with a heliocentric velocity of $v_{r}=221 \pm 30 \mathrm{~km} \mathrm{~s}^{-1}$, an effective temperature of $T_{\text {eff }}=3900_{-40}^{+60} \mathrm{~K}$ (spectral type of roughly $\mathrm{M} 0$ ), and a metallicity of $Z=-0.84_{-0.09}^{+0.10} \mathrm{dex}$ (uncertainties are singleparameter $1 \sigma$-confidence intervals based on the $\chi^{2}$ statistics after it was re-scaled to yield a reduced $\chi^{2}$ of about 1 , and the radial velocity uncertainty includes systematic uncertainties to account for the wavelength scale).

\footnotetext{
${ }^{27}$ https://github.com/ebellm/pyraf-dbsp
} 


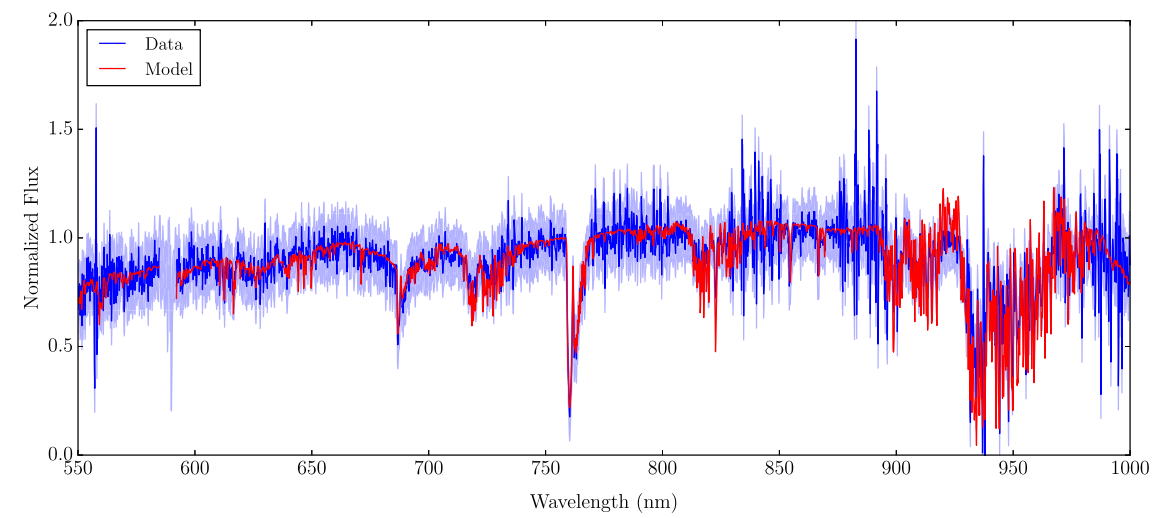

Figure 4. Normalized spectrum of 1024-Br from the Palomar 200 inch DBSP observation. The blue line is the data, with the uncertainties indicated by the shaded region. The red line is the best-fit Phoenix model with radial velocity $v_{r}=221 \mathrm{~km} \mathrm{~s}^{-1}$, effective temperature of $T_{\text {eff }}=3900 \mathrm{~K}$, and a metallicity of $Z=-0.84$ dex (the surface gravity was fixed to 4.9 dex).

\subsection{Spectral Energy Distribution}

Based on the spectroscopic result, we analyzed the spectral energy distribution using the archival FORS 1 photometry from Sutaria et al. (2003) along with the 2MASS $J$-band (the source was not detected in the $K_{s}$-band, and the $H$-band point had low $\mathrm{S} / \mathrm{N}$ ) and WISE (Wright et al. 2010) $W_{1^{-}}$and $W_{2}$-band data again using Phoenix models. For all of the observed magnitudes, a systematic uncertainty of $0.045 \mathrm{mag}$ is added in quadrature to obtain a reduced $\chi^{2}$ of about 1 at the best fit. We determined the line-of-sight reddening using the threedimensional models of Green et al. (2015), finding $E(B-V)=0.04$ mag for distances $>1 \mathrm{kpc}$ (consistent with the value used by Sutaria et al. 2003). With the metallicity and surface gravity set to the spectroscopic result, we obtain fit parameters very similar to the spectroscopic values: $T_{\text {eff }}=3874_{-29}^{+208} \mathrm{~K}$ and a distance (based on an assumed surface gravity of $\log g=4.9$ dex and mass $M=0.4 M_{\odot}$, as is appropriate for a low-metallicity star with this effective temperature; Chabrier \& Baraffe 1997) of $d=1.08 \pm 0.04 \mathrm{kpc}$, which is consistent with the radio timing. The fit is shown in Figure 5.

\section{A WIDE BINARY COMPANION?}

Following the discussions in Matthews et al. (2016) and Desvignes et al. (2016), we consider whether or not PSR J1024-0719 and 1024-Br form a binary and, if so, how we could constrain its parameters (also see Lyne et al. 2015). We have shown that the pulsar and the optical source have absolute positions consistent within uncertainties (Section 3.2). If we adopt the recent parallax distances for PSR J1024-0719 rather than the DM distance, then its distance is also consistent with the main-sequence distance for 1024-Br. Therefore, the objects appear to align in three dimensions. Since they also form a common proper motion pair, they align in two further dimensions of phase space. Could a wide binary system satisfy our further dynamical constraints? We consider three specific constraints.

1. The intrinsic period derivative of the pulsar should be $>0$, and is likely $\lesssim 10^{-19} \mathrm{~s} \mathrm{~s}^{-1}$ consistent with most MSPs.

2. The pulsar and putative companion are separated by $\lesssim 0$ !' 15 on the sky.

3. The pulsar should have a period second derivative $\ddot{P}=(3.4 \pm 0.9) \times 10^{-24} \mathrm{yr}^{-1}($ Section 2$)$.

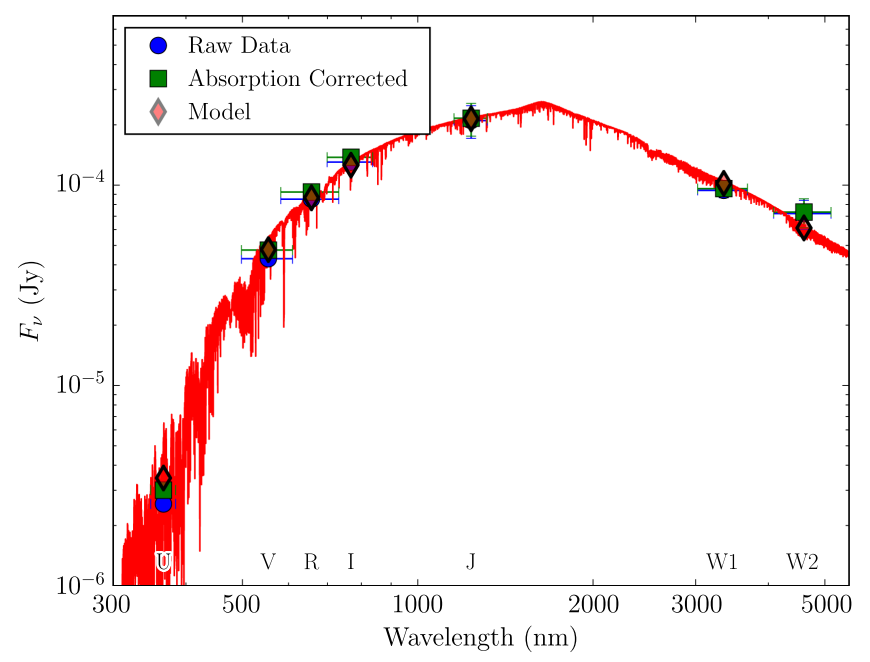

Figure 5. Spectral energy distribution (SED) of the 1024-Br. The blue circles are the raw photometry from Sutaria et al. (2003) along with archival 2MASS and WISE data, where we use the zero-point flux densities from Bessell et al. (1998), Cohen et al. (2003), and Jarrett et al. (2011). The green squares have been corrected for extinction with $E(B-V)=0.04$. The red curve is a Phoenix model atmosphere for the best-fit effective temperature of $3850 \mathrm{~K}$, and the red diamonds are that model atmosphere integrated over the filter passbands.

\subsection{Circular Orbit Models}

While there is no a priori reason to assume that the orbits of the pulsar and companion are circular, such an assumption simplifies the analysis and can elucidate the broad properties of the system. Thus, we begin by considering circular orbits; we broaden the analysis to include eccentric orbits in Section 4.2.

Constraint \#1. If we posit that the pulsar and companion are in a wide orbit such that only low-order period derivatives are apparent in the timing residuals, then we can constrain the properties of the orbit. First, we take

$$
\dot{P}_{\text {int }}=\dot{P}_{\text {obs }}-\dot{P}_{\text {Shk }}-\dot{P}_{\text {orb }}
$$

as the intrinsic $\dot{P}_{\text {int }}$, where we correct the observed $\dot{P}_{\text {obs }}$ for the Shklovskii effect, $\dot{P}_{\text {Shk }}$, and for any dynamical influence of an orbit, $\dot{P}_{\text {orb }}$. Note that $\dot{P}_{\text {orb }}$ refers to a change in the pulsar period due to orbital motion, not a change in the period of the orbit. We ignore corrections for differential acceleration in the Galactic potential, which are small for PSR J1024-0719. 


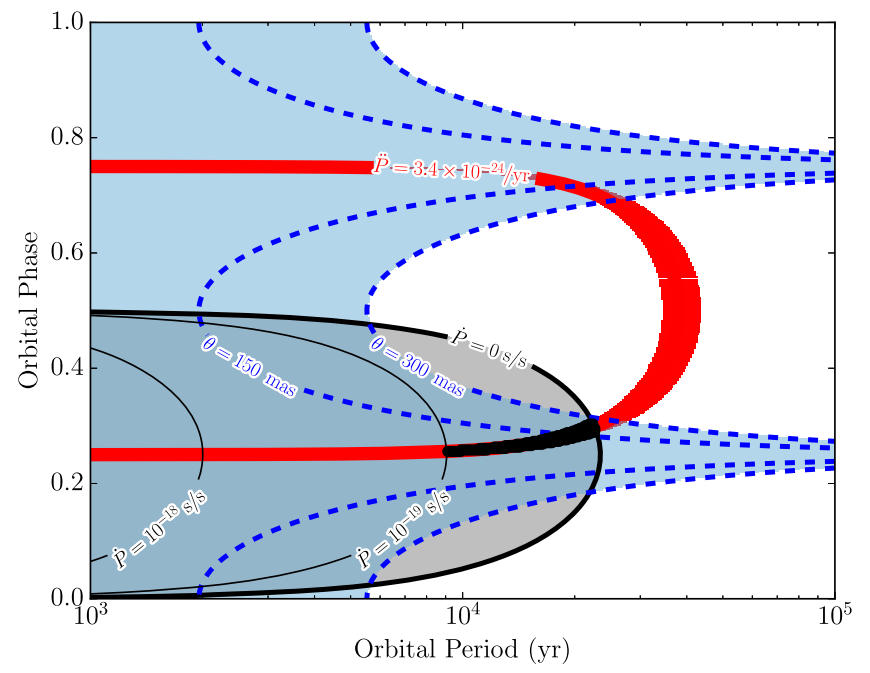

Figure 6. Constraints on the PSR J1024-0719 orbital period and orbital phase (mean anomaly) assuming a circular, edge-on orbit. The gray shaded region shows the constraint $\dot{P}_{\text {int }}<0$, and the solid black lines show constraints at specific values of $\dot{P}_{\text {int }}$. Typical millisecond pulsars have $0<\dot{P}_{\text {int }}<10^{-19} \mathrm{~s} \mathrm{~s}^{-1}$, and so the PSR J1024-0719 orbit should lie between these lines. The blue shaded region shows the constraint from angular separation on the sky, $\theta<300$ mas, and dashed blue lines show constraints at specific values of $\theta$. The red contour is where $\ddot{P}=(3.4 \pm 0.9) \times 10^{-24} \mathrm{yr}^{-1}$. The thick black region meets all of these criteria.

For a circular orbit,

$$
\begin{aligned}
\dot{P}_{\mathrm{orb}} & =\frac{G M_{\mathrm{c}} P \sin i}{a^{2} c} \sin \phi \\
& =\frac{G^{1 / 3} M_{\mathrm{c}} P \sin i}{\left(M_{\mathrm{c}}+M_{\mathrm{psr}}\right)^{2 / 3} c}\left(\frac{2 \pi}{P_{\mathrm{b}}}\right)^{4 / 3} \sin \phi,
\end{aligned}
$$

where $M_{\mathrm{c}}$ is the companion mass, $M_{\mathrm{psr}}$ is the pulsar mass, $a$ is the orbital semimajor axis (full separation between the pulsar and companion), $i$ is the inclination, $P_{\mathrm{b}}$ is the binary period, and $\phi$ is the orbital phase (mean anomaly, measured from 0 to 1 , with 0 being the ascending node). Figure 6 (black lines) shows the constraints that arise from Equations (1) and (2) for different values of $\dot{P}_{\text {int }}$, using our observed value of the companion mass, $M_{\mathrm{c}} \approx 0.4 M_{\odot}$, and assuming (for simplicity) an edge-on orbit, $i=90^{\circ}$, and a fixed pulsar mass of $M_{\mathrm{psr}}=1.54 M_{\odot}($ Özel $\&$ Freire 2016). Typical solutions have orbital periods of $P_{\mathrm{b}} \approx 10 \mathrm{kyr}$, with the maximum allowed value of $\approx 30 \mathrm{kyr}$. In order to have a positive $\dot{P}_{\text {int }}$, the pulsar must have an orbital phase of $0.0<\phi<0.5$.

Constraint \#2. We consider the projected separation between PSR J1024-0719 and the putative companion. For a wide orbit, there will be some phases where the projected separation between the pulsar and the companion is quite large. The constraints for the parameters of the PSR J1024-0719 system are shown in Figure 6 as dashed blue lines for our estimated upper limit on separation, $\theta<150$ mas (Section 3.2) and for a more conservative $\theta<300$ mas. For a circular orbit, the maximum separation is $919\left(P_{\mathrm{b}} / 20 \mathrm{kyr}\right)^{3 / 2} d_{1}$ mas at quadrature $(\phi=0$ or $\phi=0.5)$, where the distance is $1 d_{1}$ $\mathrm{kpc}$, while the minimum projected separation is $919 \cos i\left(P_{\mathrm{b}} / 20 \mathrm{kyr}\right)^{3 / 2} d_{1}$ mas at conjunction $(\phi=0.25$ or $\phi=0.75)$. So, if the pulsar and companion were near quadrature, then they would violate our limit on $\theta$ regardless of inclination, but near conjunction they can satisfy this constraint.

Constraint \#3. Finally. we consider the period second derivative. This comes from the jerk (time derivative of the acceleration) along the line of sight in the orbit. In a circular orbit, the dynamical $\ddot{P}$ is

$$
\begin{aligned}
\ddot{P}_{\mathrm{orb}} & =\frac{G^{3 / 2} M_{\mathrm{c}}\left(M_{\mathrm{c}}+M_{\mathrm{psr}}\right) P \sin i}{a^{7 / 2} c} \cos \phi \\
& =\frac{G^{1 / 3} M_{\mathrm{c}} P \sin i}{\left(M_{\mathrm{c}}+M_{\mathrm{psr}}\right)^{2 / 3} c}\left(\frac{2 \pi}{P_{\mathrm{b}}}\right)^{7 / 3} \cos \phi .
\end{aligned}
$$

The constraint based on the observed $\ddot{P}$ is shown in Figure 6 .

As seen in Figure 6, all three of these constraints are satisfied by edge-on circular binary systems with orbital periods 10-30 kyr and appropriate orbital phases. For inclined circular orbits (not shown) these constraints can still all be met. The orbital period decreases to around $2 \mathrm{kyr}$ as the inclination approaches 0 .

\subsection{Eccentric Orbit Models}

We can find solutions for the general case of inclined, eccentric orbits (based on Joshi \& Rasio 1997; Freire et al. 2001). To fully explore the phase space, we undertook a Markov-Chain Monte Carlo (MCMC) exploration of the eight-dimensional phase space. We varied the orbital period $P_{\mathrm{b}}$, inclination $i$, eccentricity $e$, distance $d$, companion mass $M_{\mathrm{c}}$, and proper motion $\mu$, along with nuisance parameters for the mean anomaly and the longitude of periastron. As in Section 4.1, for simplicity, we held the pulsar mass fixed at $1.54 M_{\odot}$; an analysis with different pulsar mass values would produce qualitatively similar results, with minor rescalings of the parameter values. We assumed prior distributions on the parallax $(\varpi=0.78 \pm 0.23$ mas $)$ and proper motion

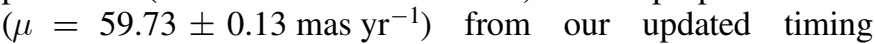
(Section 2), and $M_{\mathrm{c}}=0.4 \pm 0.1 M_{\odot}$ to match our SED fitting. We also included flat prior distributions on $\cos i$ and $\log P_{\mathrm{b}}$. The posterior was evaluated with a hard cutoff for $\dot{P}_{\text {int }}$, requiring it to be between 0 and $10^{-19} \mathrm{~s} \mathrm{~s}^{-1}$. We evaluated the goodness-of-fit by comparing the inferred $\ddot{P}$ against the measured value of $(3.4 \pm 0.9) \times 10^{-24} \mathrm{yr}^{-1}$ as well as the projected separation with a best-fit value of 0 and uncertainty of 0.' 15. Usingemcee (Foreman-Mackey et al. 2013), we used 600 "walkers" for 50,000 iterations each, starting off with the walkers randomly distributed in phase space according to the priors described above. After removing 100 iterations for "burn-in" and thinning the samples by a factor of about 1000 to account for correlations among the points, we had roughly 20,000 individual samples for each parameter. The results are shown in Figure 7. We see results broadly consistent with our inferences from the edge-on circular orbits: binary periods near $10^{4} \mathrm{yr}$ are preferred, as are edge-on orbits, and overall lower eccentricities are better but no eccentricity is excluded. There is a general covariance between $P_{\mathrm{b}}$ and $i$, with smaller periods needed at more face-on inclinations (reinforcing our results from above) but allowing larger distances, and the minimum binary periods are around $2 \mathrm{kyr}$. The lower binary periods are preferred solutions with higher eccentricities, and there is a clear selection of eccentricity based on the sign of $\dddot{P}$; if $\dddot{P}>0$, then more circular (and hence wider and more edge-on) orbits are preferred, but if $\dddot{P}<0$, then circular orbits cannot fit the 


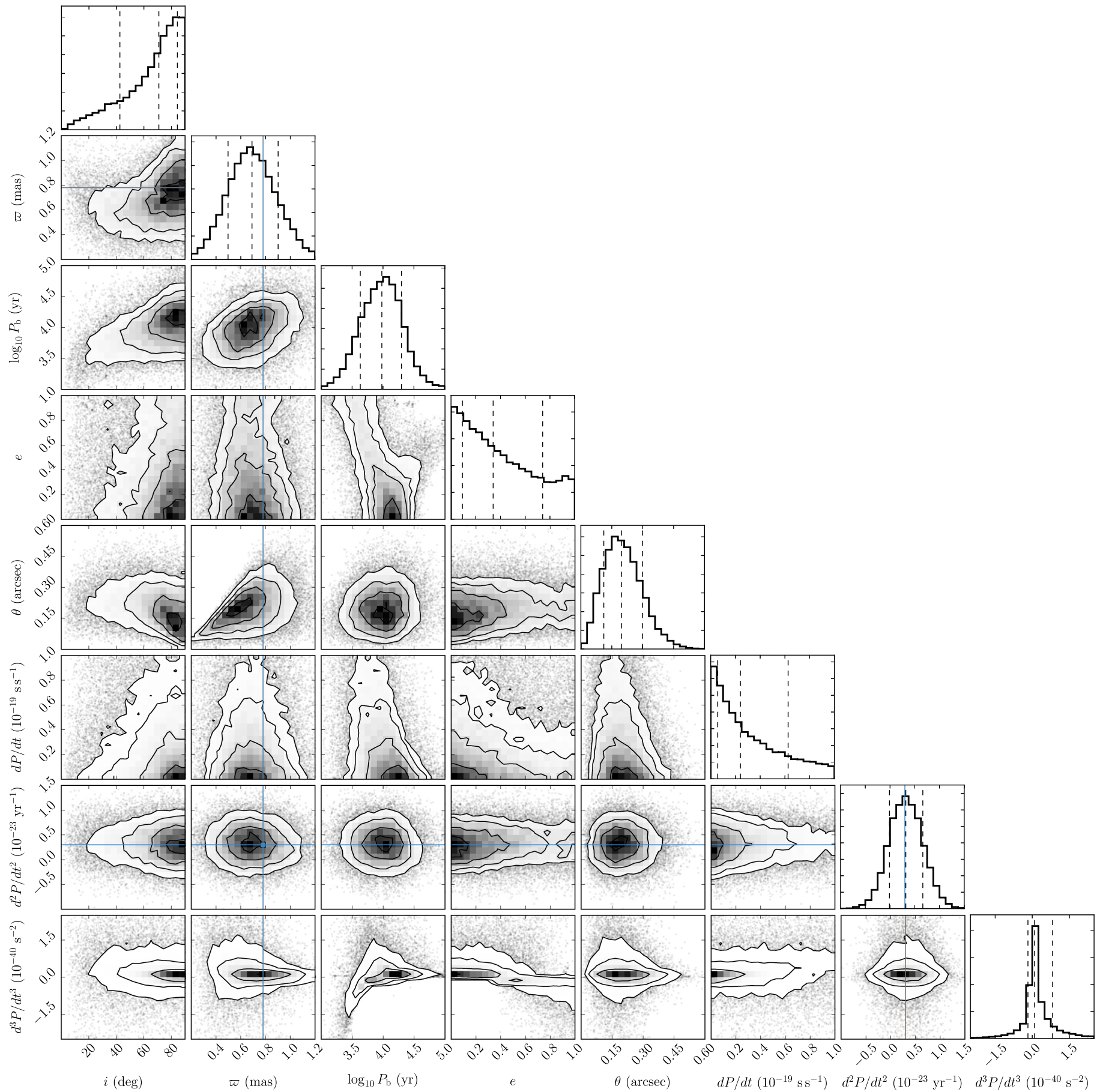

Figure 7. Markov-Chain Monte Carlo (MCMC) results for possible orbits for PSR J1024-0719. We show the joint two-dimensional contours for the inclination $i$, parallax $\varpi$, binary period $P_{\mathrm{b}}$, and eccentricity $e$, along with derived parameters $\dot{P}_{\text {int }}, \ddot{P}_{\text {orb }}$, and $\dddot{P}_{\text {orb }}$. The vertical lines show the median and $\pm 1 \sigma$ constraints on the onedimensional marginal distributions, while the contours show $0.5,1,1.5$, and $2 \sigma$ joint confidence regions. The blue vertical/horizontal lines are the measured value of $\varpi$ and $\ddot{P}$ from Section 2 .

data (following Figure 6) and we need higher eccentricities, lower $P_{\mathrm{b}}$, and more face-on orbits.

Overall, we conclude that a wide binary system is completely compatible with all of the observational constraints on PSR J1024-0719 and 1024-Br.

\section{DISCUSSION}

We now consider the implications of such a binary system for some of the puzzling measurements discussed above.
The $\gamma$-ray efficiency should be revised for the updated $\dot{P}$ and distance. The $\gamma$-ray flux is $3.8 \times 10^{-12} \mathrm{erg} \mathrm{s}^{-1} \mathrm{~cm}^{-2}$ (Espinoza et al. 2013), so the luminosity is $4.5 \times 10^{32} d_{1}^{2} \mathrm{erg} \mathrm{s}^{-1}$ (assuming beaming into $4 \pi$ ster). If $\dot{P}_{\text {int }}$ is as high as $10^{-19} \mathrm{~s} \mathrm{~s}^{-1}$, which is certainly possible (Figures 6 and 7), then this implies a spin-down luminosity as large as $\dot{E}=3.1 \times 10^{34} \mathrm{erg} \mathrm{s}^{-1}$, or a $\gamma$-ray efficiency as low as $1.5 d_{1}^{2} \%$. Likely, the true value is not this low, but this at least resolves the possible problem raised by Matthews et al. (2016) 


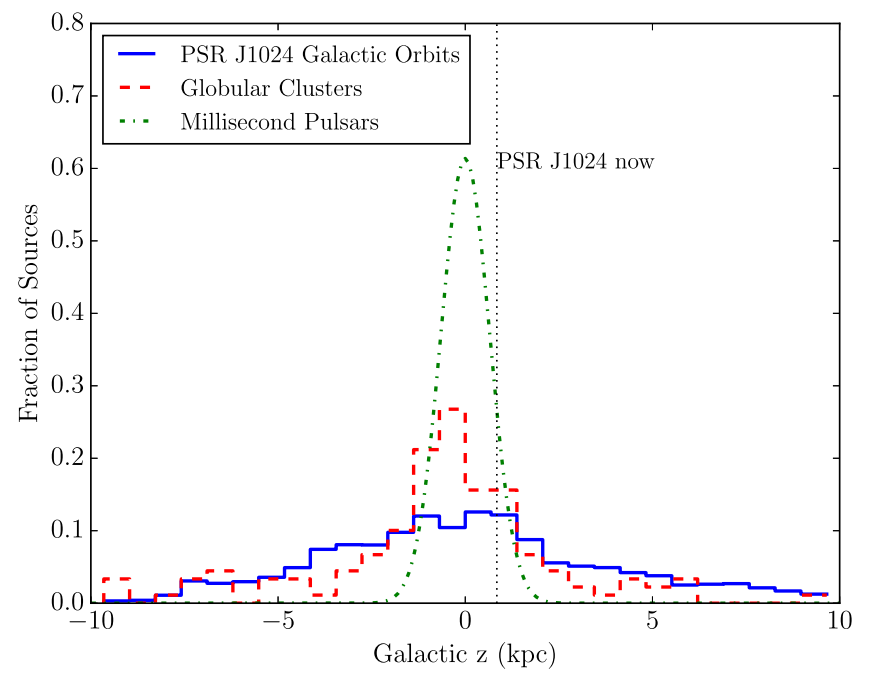

Figure 8. Distribution of the distance of PSR J1024-0719 above/below the Galactic plane $z$ (solid blue line), shown for 100 orbits in the Galactic potential over the past 1 Gyr calculated using galpy (Bovy 2015). We also compare with the distribution of Galactic globular clusters at their current positions (Harris 1996, 2010 edition; red dashed line) and the vertical distribution of MSPs from Cordes \& Chernoff (1997, green dotted-dashed line). The current $z$ of PSR J1024-0719 $(0.84 \mathrm{kpc})$ is the vertical dotted line.

regarding the apparent extremely high efficiency at the parallax distance.

Similarly, we must revise the analysis of the X-ray luminosity. Zavlin (2006) find a thermal luminosity of $2.6 \times 10^{30} d_{1}^{2} \mathrm{erg} \mathrm{s}^{-1}$. If $\dot{E}$ is as high as that in the previous analysis, then the X-ray efficiency would be as low as $10^{-4.1}$, which is somewhat lower than most objects in Zavlin (2006) but less discrepant than it was before.

While a wide binary system resolves some of the questions regarding the distance, a major remaining puzzle is its high transverse velocity, $v_{\perp}=282 d_{1} \mathrm{~km} \mathrm{~s}^{-1}$, and what that implies about the possible formation mechanisms. As discussed by Matthews et al. (2016), if placed at its parallax distance, PSR J1024-0719 has a much higher velocity than most MSPs. Using a radial velocity $v_{r}=221 \pm 30 \mathrm{~km} \mathrm{~s}^{-1}$, we find velocities $(U, V, W)=(-82 \pm 15,-436 \pm 122,-164 \pm$ 135) $\mathrm{km} \mathrm{s}^{-1}$ with respect to the Local Standard of Rest using the Solar motion from Hogg et al. (2005). This agrees roughly with the velocity ellipsoid for metal-poor halo stars (e.g., Chiba $\&$ Beers 2000, although it prefers metallicities $\lesssim-2$ dex), or with the radial (Harris 1996, 2010 edition) and tangential (e.g., Kalirai et al. 2007) velocities of globular clusters. However, it is about four times the velocity dispersion for MSPs (Cordes \& Chernoff 1997; Matthews et al. 2016), and if we integrate the orbit of PSR J1024-0719 in the Galactic potential (using galpy; Bovy 2015), then we find a scale height of 2-4 kpc (Figure 8), compared to $0.65 \mathrm{kpc}$ for MSPs (Cordes \& Chernoff 1997, also see Levin et al. 2013). This suggests that, kinematically, PSR J1024-0719 belongs to a separate population than the vast majority of MSPs, and this may relate to how it was formed.

Young pulsars with very high space velocities are known (e.g., Chatterjee et al. 2005), and they likely rely on binary disruption and/or supernova kicks for their high velocities. Similarly, hypervelocity stars (e.g., Brown et al. 2005) are often thought to originate (Tauris 2015) from binaries disrupted by a supermassive black hole (e.g., Hills 1988) or a supernova
(Blaauw 1961); other possibilities such as a tidal stream (P. Németh et al. 2016, in preparation) or dynamical ejection following an exchange in a dense stellar environment (Aarseth 1974) may also operate. However, the case of PSR J1024-0719 is different from both young pulsars and hypervelocity stars, in that it is presumably recycled following prolonged stellar evolution in a close $\left(P_{\mathrm{b}} \sim\right.$ day) binary (e.g., Tauris et al. 2012) with a companion that is now absent. Instead, its companion is anomalous, more like the eccentric binary PSR J1903+0327 (Champion et al. 2008; Freire et al. 2011). We note that estimates suggest less than $1 \%$ of the MSP population originates from the halo (Cordes \& Chernoff 1997), which could be consistent with finding a single object like PSR J1024-0719 in the 100 well-timed MSPs, but PSR J1024-0719 likely requires a denser natal environment, such as a globular cluster (see P. Németh et al. 2016, in preparation), to have had the dynamical encounters that removed the original companion and left the current one.

To further explore this topic, we compare with PSR B1620-26 (Lyne et al. 1988) in the globular cluster M4, which has a white dwarf in a 191 day orbit and a Jupiter-mass companion in a decades-long outer orbit (Thorsett et al. 1999). Most formation scenarios favor a dynamical encounter in the dense core of the globular cluster (Sigurdsson et al. 2003) which exchanged the planet into the MSP system to explain the wide eccentric orbit. Recoil following the exchange can explain why the PSR B1620-26 system is currently on the outskirts of M4 on a wide orbit in the cluster's potential, although it is still likely bound. As much as 50\% of the globular cluster MSP population could be ejected (Ivanova et al. 2008) and further objects could be tidally stripped (Gnedin \& Ostriker 1997), which could explain the origin of PSR J1024-0719 in the Galactic plane (see Champion et al. 2008).

We suggest that PSR J1024-0719 was formed in a globular cluster (which form MSPs at a very high rate due to the many stellar encounters; e.g., Sigurdsson \& Phinney 1995; Verbunt \& Freire 2014), and that its initial evolution was much like most other such systems with recycling in a compact binary with a white dwarf. A subsequent dynamical encounter with another binary (also see DeCesar et al. 2015) exchanged/ ejected the white dwarf and led to the current system. There might have also been a phase including a triple system, whose disruption might explain the very wide orbit. Eventually, either as the result of the initial encounter or subsequent encounters the PSR J1024-0719 system would have been ejected from the globular cluster (which only requires a recoil velocity of $\sim 30 \mathrm{~km} \mathrm{~s}^{-1}$, consistent with most dynamical predictions). The velocity of the system now would be the halo velocity of the cluster plus a small amount, consistent with the orbit we now see. Note that we cannot trace the system back to a potential cluster of origin given the poor knowledge of space velocities for most globular clusters and the unknown age of this system. However, the sub-solar metallicity we see for $1024-\mathrm{Br}$ is consistent with typical values for globular clusters.

Matthews et al. (2016) analyzed the MSP velocity distribution and posited a model in which the bulk of the MSP population is formed in the Galactic disk and has velocities similar to the thermal velocities of other old stellar populations, but in which there are a few high-speed outliers. Our formation scenario for PSR J1024-0719 suggests that the ejecta of globular clusters may be the source of the outlier population. 
Bassa et al. (2016) came to conclusions very similar to ours regarding the nature of the PSR J1024-0719 system using largely independent radio and optical data sets. They proposed a formation scenario in which the system is the remnant of a hierarchical triple system formed in the Galactic disk, with its high space velocity the result of a supernova kick. In both scenarios, some degree of fine tuning is required to end up with the current barely-bound binary and to match the space velocity. The true origin may be a combination of both scenarios, with a hierarchical triple evolving in a globular cluster and being ejected as it evolves into a wide MSP binary system. Such a scenario might remove some of the fine tuning needed above and in Bassa et al. (2016).

\section{CONCLUSIONS}

We have presented new radio timing along with archival optical data that strongly suggest PSR J1024-0719 is in a wide (2-20 kyr) binary orbit with a low-mass stellar companion. Our preferred formation mechanism is that the system was formed through a dynamical exchange in a globular cluster, which would explain the strange companion, the wide orbit, and the large space velocity, but this needs to be confirmed with detailed numerical experiments. The currently available radio timing data cannot determine the orbital parameters uniquely, but further observations and astrometric measurements of this system might help pin down its parameters and constrain formation scenarios.

The detection of further period derivatives is one such measurement, although care must be taken to separate dynamical period derivatives from timing noise, dispersion measure variations, and other effects. With observations made over a longer time span, the next-accessible parameter of interest is the period third derivative, $\ddot{P}$. Positive values of $\dddot{P}$ are required for circular orbits and are highly favored for elliptical orbits. As shown in Figure 7, the value should be of the order of $|\ddot{P}| \sim 1 \times 10^{-40} \mathrm{~s}^{-2}$, or $\left|f_{3}\right| \sim 4 \times 10^{-36} \mathrm{~s}^{-4}$. We estimate that such a measurement could be achieved at $3 \sigma$ significance by observations such as ours, using dual-receiver measurements with monthly cadence, made over 15 years. We emphasize that dual-receiver measurements are critical: even in the existing data set, PSR J1024-0719 shows time-variable dispersion measures more complex than a simple quadratic or cubic pattern over time, the effect of which can only be removed through observations at widely separated radio frequencies.

Additional progress will be made by GAIA (de Bruijne 2012) observations of the companion to tie its astrometry directly to the ICRF at high precision: while the distance is unlikely to be significantly refined ${ }^{28}$, the absolute astrometry will be useful.

We thank J. Creighton, C. Bassa, and S. Phinney for useful discussions. The NANOGrav project receives support from National Science Foundation (NSF) PIRE program award number 0968296 and NSF Physics Frontiers Center award number 1430284. P.S.R.'s work at NRL is supported by the Chief of Naval Research. Pulsar research at UBC is supported by an NSERC Discovery Grant and by the Canadian Institute for Advanced Research. A.A.M. acknowledges support for this

\footnotetext{
28 We predict a GAIA magnitude of $G=19.0$ (Jordi et al. 2010) for the companion, which leads to a parallax uncertainty of $300 \mu$ as (de Bruijne et al. 2014) and no radial velocity measurement. This compares with an uncertainty of $140 \mu$ as from Guillemot et al. (2016) or $230 \mu$ as from Table 2.
}

work by NASA from a Hubble Fellowship grant: HST-HF51325.01, awarded by STScI, operated by AURA, Inc., for NASA, under contract NAS 5-26555. Part of the research was carried out at the Jet Propulsion Laboratory, California Institute of Technology, under a contract with NASA. The National Radio Astronomy Observatory is a facility of the National Science Foundation operated under cooperative agreement by Associated Universities, Inc.

Facilities: GBT, PO:1.5m, Hale (Double Beam Spectrograph), VLT:Antu (FORS1).

\section{REFERENCES}

Aarseth, S. J. 1974, A\&A, 35, 237

Arzoumanian, Z., Brazier, A., Burke-Spolaor, S., et al. 2015, ApJ, 813, 65

Bailes, M., Johnston, S., Bell, J. F., et al. 1997, ApJ, 481, 386

Bassa, C. G., Janssen, G. H., Stappers, B. W., et al. 2016, MNRAS, submitted Bertin, E., \& Arnouts, S. 1996, A\&AS, 117, 393

Bessell, M. S., Castelli, F., \& Plez, B. 1998, A\&A, 333, 231

Blaauw, A. 1961, BAN, 15, 265

Bovy, J. 2015, ApJS, 216, 29

Brown, W. R., Geller, M. J., Kenyon, S. J., \& Kurtz, M. J. 2005, ApJL, 622, L33

Caballero, R. N., Lee, K. J., Lentati, L., et al. 2015, arXiv:1510.09194

Cenko, S. B., Fox, D. B., Moon, D.-S., et al. 2006, PASP, 118, 1396

Chabrier, G., \& Baraffe, I. 1997, A\&A, 327, 1039

Champion, D. J., Ransom, S. M., Lazarus, P., et al. 2008, Sci, 320, 1309

Chatterjee, S., Vlemmings, W. H. T., BrisAken, W. F., et al. 2005, ApJL, 630, L61

Chiba, M., \& Beers, T. C. 2000, AJ, 119, 2843

Cohen, M., Wheaton, W. A., \& Megeath, S. T. 2003, AJ, 126, 1090

Cordes, J. M., \& Chernoff, D. F. 1997, ApJ, 482, 971

Cordes, J. M., \& Lazio, T. J. W. 2002, arXiv:astro-ph/0207156

de Bruijne, J. H. J. 2012, Ap\&SS, 341, 31

de Bruijne, J. H. J., Rygl, K. L. J., \& Antoja, T. 2014, EAS Publications Ser., 67,23

DeCesar, M. E., Ransom, S. M., Kaplan, D. L., Ray, P. S., \& Geller, A. M. 2015, ApJL, 807, L23

Desvignes, G., Caballero, R. N., Lentati, L., et al. 2016, MNRAS, 458, 3341 Espinoza, C. M., Guillemot, L., Çelik, Ö, et al. 2013, MNRAS, 430, 571

Folkner, W. M., Williams, J. G., Boggs, D. H., Park, R. S., \& Kuchynka, P. 2014, IPNPR, 196, 1

Foreman-Mackey, D., Hogg, D. W., Lang, D., \& Goodman, J. 2013, PASP, 125,306

Freire, P. C., Kramer, M., \& Lyne, A. G. 2001, MNRAS, 322, 885

Freire, P. C. C., Bassa, C. G., Wex, N., et al. 2011, MNRAS, 412, 2763

Gnedin, O. Y., \& Ostriker, J. P. 1997, ApJ, 474, 223

Green, G. M., Schlafly, E. F., Finkbeiner, D. P., et al. 2015, ApJ, 810, 25

Guillemot, L., Smith, D. A., Laffon, H., et al. 2016, A\&A, 587, 109

Harris, W. E. 1996, AJ, 112, 1487

Hills, J. G. 1988, Natur, 331, 687

Hogg, D. W., Blanton, M. R., Roweis, S. T., \& Johnston, K. V. 2005, ApJ, 629,268

Horne, K. 1986, PASP, 98, 609

Hotan, A. W., Bailes, M., \& Ord, S. M. 2006, MNRAS, 369, 1502

Husser, T.-O., Wende-von Berg, S., Dreizler, S., et al. 2013, A\&A, 553, A6

Ivanova, N., Heinke, C. O., Rasio, F. A., Belczynski, K., \& Fregeau, J. M. 2008, MNRAS, 386, 553

Jarrett, T. H., Cohen, M., Masci, F., et al. 2011, ApJ, 735, 112

Jordi, C., Gebran, M., Carrasco, J. M., et al. 2010, A\&A, 523, A48

Joshi, K. J., \& Rasio, F. A. 1997, ApJ, 479, 948

Kalirai, J. S., Anderson, J., Richer, H. B., et al. 2007, ApJL, 657, L93

Levin, L., Bailes, M., Barsdell, B. R., et al. 2013, MNRAS, 434, 1387

Lyne, A. G., Biggs, J. D., Brinklow, A., McKenna, J., \& Ashworth, M. 1988, Natur, 332, 45

Lyne, A. G., Stappers, B. W., Keith, M. J., et al. 2015, MNRAS, 451, 581

Matthews, A. M., Nice, D. J., Fonseca, E., et al. 2016, ApJ, 818, 92

Moehler, S., Modigliani, A., Freudling, W., et al. 2014, A\&A, 568, A9

Oke, J. B., \& Gunn, J. E. 1982, PASP, 94, 586

Özel, F., \& Freire, P. 2016, ARA\&A, in press (arXiv:1603.02698)

Qi, Z., Yu, Y., Bucciarelli, B., et al. 2015, AJ, 150, 137

Reardon, D. J., Hobbs, G., Coles, W., et al. 2016, MNRAS, 455, 1751

Roeser, S., Demleitner, M., \& Schilbach, E. 2010, AJ, 139, 2440

Shannon, R. M., \& Cordes, J. M. 2010, ApJ, 725, 1607 
Shklovskii, I. S. 1970, SvA, 13, 562

Sigurdsson, S., \& Phinney, E. S. 1995, ApJS, 99, 609

Sigurdsson, S., Richer, H. B., Hansen, B. M., Stairs, I. H., \& Thorsett, S. E. 2003, Sci, 301, 193

Skrutskie, M. F., Cutri, R. M., Stiening, R., et al. 2006, AJ, 131, 1163

Smiljanic, R., Korn, A. J., Bergemann, M., et al. 2014, A\&A, 570, A122

Sutaria, F. K., Ray, A., Reisenegger, A., et al. 2003, A\&A, 406, 245

Tauris, T. M. 2015, MNRAS, 448, L6

Tauris, T. M., Langer, N., \& Kramer, M. 2012, MNRAS, 425, 1601
Thorsett, S. E., Arzoumanian, Z., Camilo, F., \& Lyne, A. G. 1999, ApJ, 523,763

Toscano, M., Sandhu, J. S., Bailes, M., et al. 1999, MNRAS, 307, 925

Verbiest, J. P. W., Weisberg, J. M., Chael, A. A., Lee, K. J., \& Lorimer, D. R. 2012, ApJ, 755, 39

Verbunt, F., \& Freire, P. C. C. 2014, A\&A, 561, A11

Vickers, J. J., Roeser, S., \& Grebel, E. K. 2016, AJ, 151, 99

Wright, E. L., Eisenhardt, P. R. M., Mainzer, A. K., et al. 2010, AJ, 140, 1868

Zavlin, V. E. 2006, ApJ, 638, 951 